\title{
Prognostic clinical factors in patients affected by non-small-cell lung cancer receiving Nivolumab
}

Francesco Pantano, Marco Russano, Alfredo Berruti, Giovanni Mansueto, Maria R. Migliorino, Vincenzo Adamo, Giuseppe Aprile, Alain Gelibter, Corrado Ficorella, Alfredo Falcone, Antonio Russo, Michele Aieta, Michele Maio, Olga Martelli, Sandro Barni, Andrea Napolitano, Elisa Roca, Silvia Quadrini, Daniela lacono, Alessandro Russo, Lorenzo Calvetti, Mario A. Occhipinti, Alessio Cortellini, Enrico Vasile, Francesco Passiglia, Marco Imperatori, Luana Calabrò, Anna M. Di Giacomo, Fausto Petrelli, Giulia Pasquini, Tindara Franchina, Olga Venditti, Salvatore Intagliata, Antonio Galvano, Iacopo Fioroni, Bruno Vincenzi, Giuseppe Tonini \& Daniele Santini

To cite this article: Francesco Pantano, Marco Russano, Alfredo Berruti, Giovanni Mansueto, Maria R. Migliorino, Vincenzo Adamo, Giuseppe Aprile, Alain Gelibter, Corrado Ficorella, Alfredo Falcone, Antonio Russo, Michele Aieta, Michele Maio, Olga Martelli, Sandro Barni, Andrea Napolitano, Elisa Roca, Silvia Quadrini, Daniela lacono, Alessandro Russo, Lorenzo Calvetti, Mario A. Occhipinti, Alessio Cortellini, Enrico Vasile, Francesco Passiglia, Marco Imperatori, Luana Calabrò, Anna M. Di Giacomo, Fausto Petrelli, Giulia Pasquini, Tindara Franchina, Olga Venditti, Salvatore Intagliata, Antonio Galvano, lacopo Fioroni, Bruno Vincenzi, Giuseppe Tonini \& Daniele Santini (2020): Prognostic clinical factors in patients affected by non-small-cell lung cancer receiving Nivolumab, Expert Opinion on Biological Therapy, DOI: 10.1080/14712598.2020.1724953

To link to this article: https://doi.org/10.1080/14712598.2020.1724953

View supplementary material ¿

Accepted author version posted online: 03 Feb 2020. Published online: 06 Feb 2020.

Submit your article to this journal

Џ Article views: 8

Q View related articles $\sqsubset$ 
(2)

Full Terms \& Conditions of access and use can be found at https://www.tandfonline.com/action/journallnformation?journalCode=iebt20 


\section{Prognostic clinical factors in patients affected by non-small-cell lung cancer receiving Nivolumab}

Francesco Pantano ${ }^{a}$, Marco Russano ${ }^{a}$, Alfredo Berrutib , Giovanni Mansuetoc, Maria R. Migliorinod, Vincenzo Adamoe, Giuseppe Aprile ${ }^{f, g}$, Alain Gelibter ${ }^{h}$, Corrado Ficorella ${ }^{i, j}$, Alfredo Falcone $^{k}$, Antonio Russol, Michele Aietam, Michele Maion, Olga Martelli ${ }^{\mathrm{n}}$, Sandro Barni ${ }^{p}$, Andrea Napolitano ${ }^{\mathrm{a}}$, Elisa Roca ${ }^{\mathrm{b}}$, Silvia Quadrinic, Daniela lacono ${ }^{\mathrm{d}}$, Alessandro Russo ${ }^{e}$, Lorenzo Calvettif, Mario A. Occhipinti ${ }^{h}$, Alessio Cortellini $\mathbb{1}^{i, j}$, Enrico Vasile ${ }^{k}$, Francesco Passiglia', Marco Imperatori ${ }^{m}$, Luana Calabròn ${ }^{n}$ Anna M. Di Giacomon ${ }^{n}$, Fausto Petrelli ${ }^{p}$, Giulia Pasquini ${ }^{k}$, Tindara Franchina ${ }^{e}$, Olga Venditti ${ }^{i, j}$, Salvatore Intagliata ${ }^{b}$, Antonio Galvano', lacopo Fioroni ${ }^{a}$, Bruno Vincenzi ${ }^{\mathrm{a}}$, Giuseppe Tonini ${ }^{\mathrm{a}}$ and Daniele Santini ${ }^{a}$

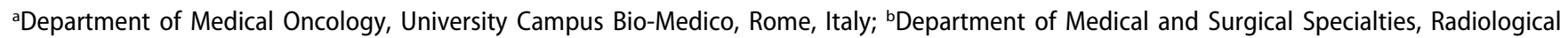
Sciences, and Public Health, University of Brescia, Medical Oncology, ASST Spedali Civili, Brescia, Italy; 'Medical Oncology Unit, ASL Frosinone,

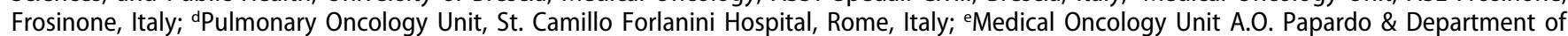
Human Pathology, University of Messina, Messina, Italy; 'Department of Oncology, University and General Hospital, Udine, Italy; ${ }^{9}$ Department of Oncology, San Bortolo General Hospital, ULSS8 Berica, Vicenza, Italy; ${ }^{\mathrm{h} M e d i c a l ~ O n c o l o g y ~ U n i t, ~ P o l i c l i n i c o ~ U m b e r t o ~ I, ~ R o m e, ~ I t a l y ; ~}{ }^{\mathrm{M} M e d i c a l}$ Oncology Unit, St. Salvatore Hospital, L'Aquila, Italy; 'Department of Biotechnological and Applied Clinical Sciences, University of L'Aquila, L'Aquila, Italy; kUnit of Oncology 2, Azienda Ospedaliero-Universitaria Pisana, Pisa, Italy; 'Section of Medical Oncology, Department of Surgical and Oncological Sciences, University of Palermo, Palermo, Italy; 'mivision of Medical Oncology, Department of Onco-Hematology, IRCCS-CROB, Referral Cancer Center of Basilicata, Rionero, Vulture, Italy; "Department of Oncology, Center for Immuno-Oncology, Medical Oncology and Immunotherapy, University Hospital of Siena, Siena, Italy; ${ }^{\circ}$ Medical Oncology Unit, Azienda Ospedale San Giovanni Addolorata, Roma, Italy; ${ }^{\mathrm{p} D i v i s i o n}$ of Oncology, Azienda Ospedaliera Treviglio, Treviglio, Italy

\section{ABSTRACT}

Background: Immune-checkpoint inhibitors have radically changed the treatment landscape of NonSmall-Cell Lung Cancer (NSCLC). It is still unclear whether specific clinical characteristics might identify those patients benefiting from immunotherapy more than others. The aim of this study was to identify clinical characteristics associated with disease-specific survival (DSS), time-to-treatment failure (TTF), objective responses (OR) and progressive disease (PD) in NSCLC patients treated with Nivolumab. Methods: This was a multicenter retrospective study conducted on 294 patients treated with Nivolumab for advanced NSCLC.

Results: Of the more than 50 variables analyzed, five showed a significant correlation with DSS: ECOG PS, size of the biggest brain metastasis, number of metastatic sites, toxicity, and malignant pleural effusion. Three variables significantly correlated with TTF: malignant pleural effusion, number of metastatic sites, number of liver metastases. Malignant pleural effusion was the only variable showing a significant correlation with OR, as well as the only one correlating with all the endpoints of the study.

Conclusions: This study identified clinical characteristics associated with survival and response during treatment with Nivolumab in NSCLC patients. The unfavorable association between malignant pleural effusion and objective response is a novel finding with important translational implications.
ARTICLE HISTORY

Received 29 July 2019

Accepted 29 January 2020

\section{KEYWORDS}

Immunotherapy; Nivolumab; non-Small-Cell Lung Cancer; malignant pleural effusion

\section{Introduction}

Lung cancer is the leading cause of cancer deaths worldwide. The two main types of lung cancer are small cell lung cancer (SCLC) and non-SCLC (NSCLC). NSCLC accounts for more than $80 \%$ of all cases [1]. In the last years, immune-checkpoint inhibitors have radically changed the treatment landscape for NSCLC patients [2]. Nivolumab, an IgG4 monoclonal antibody targeting Programmed cell Death protein 1 (PD-1), was the first immunecheckpoint inhibitor approved for the treatment of patients with advanced NSCLC experiencing progressive disease after standard platinum-based chemotherapy $[3,4]$. Recent data have shown long-lasting benefit from Nivolumab, albeit in a minority of patients [5]. This finding is similar to what previously observed in advanced melanoma patients [6,7].

It is still unclear which specific subset of patients significantly benefit from immunotherapy. Hence, there is an urgent need to identify markers predictive of response to immune-checkpoints inhibitors. To date, the level of expression of Programmed DeathLigand 1 (PD-L1) on tumor cells is the only predictive biomarker validated in the clinical practice, based on its correlation with the efficacy of Pembrolizumab, the other approved anti-PD-1 antibody $[8,9]$. Conversely, data on the efficacy of Nivolumab in presence of different levels of PD-L1 expression are controversial

CONTACT Marco Russano m.russano@unicampus.it E Department of Medical Oncology, University Campus Bio-Medico, Via Alvaro Del Portillo 200, Rome 00128, Italy

(1) Supplemental data for this article can be accessed here.

(c) 2020 Informa UK Limited, trading as Taylor \& Francis Group 
$[10,11]$. Emerging and promising biomarkers, in particular, the tumor mutation burden (TMB), are being developed and validated, especially in the setting of combination strategies including two immune-checkpoints inhibitors or an immunological agent with a chemotherapeutic one [12-14].

However, the large-scale applicability and the costeffectiveness of biological surrogates of response have to be carefully considered before implementation, especially in countries where costs are largely covered by the National Health Service. On the other hand, clinical surrogates of response have the important advantage to be readily available in any center at no additional costs. Additionally, the identification of clinical markers of response might lead to the generation of novel hypotheses on the mechanisms of action and resistance to immune-checkpoints inhibitors.

Thus, the aim of this study was to identify the clinical characteristics associated with disease-specific survival (DSS), time-to-treatment failure (TTF), objective responses (OR), and progressive disease (PD) in NSCLC patients treated with Nivolumab.

\section{Methods}

This is an exploratory, multicenter, and retrospective study conducted on 294 patients from 14 Italian centers, treated with Nivolumab for advanced NSCLC after progression to chemotherapy.

The study was approved by the Ethics Committee of the coordinator center (University Campus Bio-Medico, Rome; protocol number 23/18) and was conducted in accordance with Good Clinical Practice. The treatment period under analysis spanned from April, $1^{\text {st }} 2015$ to May, $31^{\text {th }} 2018$, data collection cutoff date. Eligibility was restricted to adult patients (aged 18 years and over) with histologically proven NSCLC, receiving Nivolumab treatment for advanced disease after at least one previous chemotherapy treatment according to regulatory approvals. Previous enrollment in clinical trials and further treatment with immune-checkpoints inhibitors were exclusion criteria. Written consent was provided by alive patients. Patients deceased for other causes rather than lung cancer were excluded. Only patients who had received at least four administrations of Nivolumab and who had performed at least one assessment evaluation scan were considered eligible. RECIST criteria were used to evaluate the radiological response to treatment. Therefore, we included only patients with measurable disease at baseline and who performed scan assessments by computed tomography (CT), magnetic resonance imaging (MRI) or positron emission tomography/computed tomography (PET/CT).

Malignant pleural effusion was defined as one of the following: 1) effusion in presence of positive pleural biopsy; 2) presence of cancerous cells in the cytological analysis of pleural fluid; 3) effusion with CT findings suggestive of malignancy (concomitant pleural nodularity, pleural rind, mediastinal pleural involvement, and pleural thickening greater than $1 \mathrm{~cm}$ ).

All patients received $3 \mathrm{mg} / \mathrm{kg}$ Nivolumab administered intravenously every 2 weeks. No patient received a flat dose of $240 \mathrm{mg}$ every 2 weeks because this schedule was approved after data collection cutoff date.
We analyzed the association of over 50 clinical variables (listed in Appendix 1) with survival and response outcomes.

\subsection{Statistical analysis}

Descriptive analyses were performed to assess incidence data. The Kaplan-Meier method was used to estimate survival intervals [15]. Survival curves were compared using the Log-Rank test [16]. All variables were evaluated as possible predictive factors for DSS and TTF using Cox model analysis. All variables were also analyzed using logistic regression to identify factors that correlated with objective responses or progressive disease as best response to Nivolumab treatment. Statistical analysis was conducted using SPSS software (version 20.00, SPSS, Chicago, IL) and Pomelo II suite (http://pomelo2.iib.uam.es). FDR-adjusted p-values $<0.05$ were considered statistically significant.

\section{Results}

\subsection{Descriptive analysis}

The study was conducted on a total of 294 patients. At the time of data collection, 153 patients were dead (52\%); of the 141 alive patients $(48 \%), 88(30 \%)$ were continuing treatment with Nivolumab. The median age at the beginning of treatment with Nivolumab was 67 years (range 34-90 years). Most patients were males (201 males, 68\%; 93 females, 32\%). The vast majority of patients had a good baseline ECOG Performance Status (ECOG-PS): 152 patients had ECOG PS 0 (52\%), 131 patients had PS 1 (44\%). Only 11 patients had PS 2 (4\%), no patient had PS 3. Smoking habit was present in most cases: 78 patients were current smokers (27\%), 175 patients were former smokers (59\%), only 30 patients were nonsmokers (10\%), smoking habit was unknown in 11 cases (4\%). The most common histology was adenocarcinoma that occurred in 54\% of cases (158 patients), followed by squamous tumor (125 patients, 42\%). Driver mutations were found in 11 patients: 10 patients with EGFR sensitizing-mutations ( 9 adenocarcinomas and 1 squamous tumor); one patient with ALK-positive adenocarcinoma. PD-L1 expression on tumor cells was detected only in 44 cases (15\%): PD-L1 expression in at least $50 \%$ of the tumor cells was reported in 9 patients, expression ranging from $1 \%$ to $49 \%$ in 19 cases, the remaining 16 patients were PD-L1 negative.

Overall, $52 \%$ of the entire study population (154 patients) received two lines of systemic treatment for metastatic disease, 29\% (84 patients) three lines, 19\% (56 patients) more than three lines. Platinum-based chemotherapy was the most widely used first-line treatment for metastatic disease (262 patients, 89\%). Nivolumab was most frequently used after progression to the first-line of treatment (190 patients, 65\%), in $23 \%$ of cases it was used in the third line (68 patients), in the remaining $12 \%$ in subsequent lines (36 patients). Docetaxel was preferred to Nivolumab in second line in $15 \%$ of cases (49 patients). Thirty-two patients (11\%) had only one disease site when they started treatment with Nivolumab. One hundred twenty-one patients (41\%) had two disease sites, 93 patients (32\%) had three sites, and the remaining 48 (16\%) had at least four sites. Nodes and lungs were the most 
common metastatic sites with a prevalence of $78 \%$ and $67 \%$, respectively. Malignant pleural effusion and bone metastases occurred in $24 \%$ of cases each. Brain, liver, and adrenal metastases were present in $14 \%, 12 \%$, and $12 \%$ of cases, respectively. Soft tissue $(3 \%)$ and peritoneal $(<2 \%)$ metastases were rare.

One hundred seven patients (36\%) experienced at least one immune-related adverse event (IrAE). Severe adverse events were reported in 28 cases (grading $\geq$ G3), and among these, 21 patients $(7 \%)$ had to permanently cease treatment. None of the study patients died due to toxicity. The most common IrAE was colitis (13\%), followed by endocrinologic toxicity (11\%), skin rash (8\%), and interstitial pneumonia (7\%). Arthritis (4\%), liver, and renal toxicities (2\%) were less common.

According to the RECIST criteria, best response to Nivolumab was complete response in 3 cases (1\%), partial response in 99 patients (34\%), stable disease in 88 cases $(30 \%)$, progressive disease in the remaining 104 (35\%). Two hundred five patients (70\%) reported clinical benefit from immunotherapy. Sixty patients were treated beyond progression (20\%) but pseudoprogression was documented in only $8 \%$ of cases (24 patients).

All the demographics and clinical parameters are listed in Table 1.

\subsection{Prognostic factors: correlation with DSS and TTF}

\subsubsection{DSS analysis}

The median Disease-Specific Survival (mDSS) of the entire study population from the start of Nivolumab treatment was 14 months (range 1 - not reached). Five variables showed statistical significance in the multivariate analysis: ECOG PS, number of metastatic sites, size of the biggest brain metastasis, toxicity, and malignant pleural effusion (Figure 1a).

Patients with poor performance status had a worse prognosis. mDSS of patients with good performance status (ECOG PS 0) was 20 months, 8 and 9 months longer than that of patients with PS ECOG 1 and PS ECOG 2, respectively (p-value 0.0004) (Figure 2a).

Two tumor burden surrogates significantly correlated with prognosis: number of metastatic sites and size of the biggest brain metastasis. Survival time decreased as the number of metastatic sites increased, passing from mDSS of 16 months in case of 1-2 metastatic sites to a median value of only 3 months in the case of 6 sites ( $p$-value <0.0001) (Figure 2b). Survival also decreased as the size of the biggest encephalic lesion increased. Considering this parameter as a continuous variable, mDSS was lower among patients with greater brain lesions ( $p$-value 0.0032). mDSS among patients with brain metastases bigger than $1 \mathrm{~cm}$ (median value in our study population, considering a value of $0 \mathrm{~cm}$ patients with no evidence of brain metastases) was significantly lower than among those with smaller brain metastases (mDSS 6 months versus 15 months; $p$-value 0.0062 ) (Figure $2 c$ ). No other tumor burden surrogate was found to significantly correlate with mDSS in the multivariate analysis.

Toxicity played a positive prognostic role: mDSS was longer among patients with at least one immune-related adverse event than among those who did not experience it (mDSS
16 months versus 12 months; p-value 0.0009) (Figure 2d). Sitespecific toxicity and the severity of the adverse event did not retain statistical significance in the multivariate analysis, while showing a positive trend with DSS.

Pleura was the only metastatic site that significantly correlated with survival. Patients with malignant pleural effusion had a mDSS of 9 months versus 16 months for patients without it ( $p$-value 0.0001) (Figure 2e).

No other variable considered in our study significantly correlated with DSS, including histology, mutational profile, smoking habit, and previous treatments (systemic treatments and radiotherapy).

\subsubsection{TTF analysis}

Three parameters significantly correlated with TTF: malignant pleural effusion, number of metastatic sites, and number of liver metastases. TTF was lower in patients with pleural effusion and/or with higher number of metastatic sites and liver metastases (Figure 1b).

\subsection{Predictive factors: correlation with $O R$ and $P D$}

The objective response (OR) and disease control (DC) rates were $34 \%$ and $65 \%$, respectively. Thirty-five percent of patients did not respond to Nivolumab. Logistic regression analysis showed that pleural effusion was the only variable significantly associated ( $p$-value 0.04 ) with OR (partial response, PR, or complete response, $C R$ ) (Figure 3a). Indeed, the presence of pleural effusion was strongly associated with a reduction of OR occurrence in different metastatic sites (Appendix 1).

On the other hand, eight variables showed a significant correlation with PD as the best response to Nivolumab treatment. Five of them correlated directly with PD: malignant pleural effusion, number of metastatic sites, ascites, number of liver metastases, and sum of the largest diameters of the biggest target lesions in each site. The other three were inversely related to PD: toxicity (at least one IrAE), age, and endocrine toxicity (Figure $3 \mathrm{~b}$ ).

Malignant pleural effusion was the only variable that showed a statistically significant correlation with all the endpoints of this survey.

Table 2 shows all factors significantly associated with the study end points (for full analysis see Appendix 1).

\section{Discussion}

In this study, we collected a large amount of real-life data on the treatment of NSCLC with Nivolumab. The large sample size and the high number of variables analyzed allowed the identification of clinical characteristics that correlated with the survival and effectiveness of Nivolumab in NSCLC patients, some of which never investigated before.

A poor ECOG PS correlated with a worse prognosis. Therefore, performance status, which is considered a key prognostic factor, retains its important clinical value also in the case of therapy with immune-checkpoints inhibitors.

Similarly, brain metastases are known negative prognostic factor. Interestingly, in our study the dimension of the biggest lesion rather than the number of metastases showed 
Table 1. Demographics and clinical parameters.

\begin{tabular}{|c|c|c|c|}
\hline General characteristics & $\mathrm{N}^{\circ}(\%)$ & Treatment with nivolumab & $\mathrm{N}^{\circ}(\%)$ \\
\hline Gender & & Line of treatment & \\
\hline Men & $201(68)$ & 2nd line & $190(65)$ \\
\hline \multirow[t]{2}{*}{ Women } & $93(32)$ & 3rd line & $68(23)$ \\
\hline & & Over 3rd-line & $36(12)$ \\
\hline \multicolumn{4}{|l|}{ ECOG PS } \\
\hline 0 & $152(52)$ & Clinical benefit & $205(70)$ \\
\hline 1 & $131(44)$ & Treatment beyond progression & $60(20)$ \\
\hline 2 & $11(4)$ & Pseudoprogression & $24(8)$ \\
\hline Smoking habit & & Best Response (RECIST) & \\
\hline Never smokers & $30(10)$ & $\mathrm{CR}$ & $3(1)$ \\
\hline Former smokers & $175(59)$ & PR & 99 (34) \\
\hline Current smokers & 78 (27) & SD & $88(30)$ \\
\hline Unknown & $11(4)$ & PD & $104(35)$ \\
\hline Histology & & IrAE & \\
\hline Adenocarcinoma & $158(54)$ & At least one IrAE & $107(36)$ \\
\hline Squamous cell carcinoma & $125(42)$ & IrAE G $\geq 3$ & $28(9)$ \\
\hline Large cell carcinoma & $1(<1)$ & Treatment discontinuation & $21(7)$ \\
\hline \multirow[t]{2}{*}{ NOS } & $10(3)$ & Skin toxicity & $24(8)$ \\
\hline & & Pneumonitis & $22(7)$ \\
\hline Mutational profile & & Colitis & $37(13)$ \\
\hline ALK re-arrengement & $1(<1)$ & Endocrinopathy & $33(11)$ \\
\hline EGFR sensitizing mutations & $10(3)$ & Arthritis & $13(4)$ \\
\hline PDL-1 expression & $44(15)$ & Nephrotoxicity & $5(2)$ \\
\hline Unknown & $250(85)$ & Liver toxicity & $5(2)$ \\
\hline Negative & $16(5)$ & & \\
\hline $1-49 \%$ & $19(7)$ & Number of metastatic sites & \\
\hline \multirow[t]{2}{*}{$\geq 50 \%$} & $9(3)$ & 1 & $32(11)$ \\
\hline & & 2 & $121(41)$ \\
\hline Stage at diagnosis & & 3 & $93(32)$ \\
\hline$|-| \mid$ & $31(11)$ & $\geq 4$ & $48(16)$ \\
\hline Illa & $24(8)$ & & \\
\hline Illb & 46 (15) & Lung metastases & $198(67)$ \\
\hline \multirow[t]{2}{*}{ IV } & $192(65)$ & $\leq 5$ & $135(46)$ \\
\hline & & $>5$ & $63(21)$ \\
\hline Treatment for metastatic disease & & Nodes metastases & $231(78)$ \\
\hline Two lines & $154(52)$ & $\leq 5$ & $167(57)$ \\
\hline Three lines & $84(29)$ & $>5$ & $64(21)$ \\
\hline \multirow{2}{*}{ More than three lines } & $56(19)$ & Liver metastases & $34(12)$ \\
\hline & & $\leq 5$ & $26(9)$ \\
\hline Palliative radiotherapy & $122(41)$ & $>5$ & $8(3)$ \\
\hline Brain & 35 (12) & Brain metastases & $42(14)$ \\
\hline Lung or mediastinal nodes & $55(19)$ & $\leq 5$ & $35(12)$ \\
\hline Adrenal gland & $2(<1)$ & $>5$ & $7(2)$ \\
\hline \multirow[t]{2}{*}{ Bone } & $36(12)$ & Bone metastases & $70(24)$ \\
\hline & & $\leq 5$ & $22(8)$ \\
\hline Timing radiotherapy & & $>5$ & $48(16)$ \\
\hline Pre-Nivolumab & $83(28)$ & Adrenal metastases & $35(12)$ \\
\hline Concomitant to Nivolumab & $23(8)$ & Soft tissue metastases & $9(3)$ \\
\hline \multirow[t]{2}{*}{ Post-Nivolumab } & $16(5)$ & Peritoneum/ascites & $5(<2)$ \\
\hline & & Malignant pleural effusion & $71(24)$ \\
\hline
\end{tabular}

a significant correlation with DSS. The number of metastatic sites, another surrogate of tumor burden, also correlated with both DSS and TTF. Furthermore, this was the variable with the highest statistical significance in the correlation with PD as the best response, suggesting that the high tumor burden correlated both with a worse prognosis and a poor efficacy of Nivolumab. This assumption is supported by other findings, such as the number of liver metastases correlating with both TTF and PD as best response, and the sum of the largest diameters of the target lesions also correlating with PD as best response.

Another relevant finding is the positive role played by toxicity. The presence of at least one IrAE, regardless of the type and severity of the event, correlated with a better DSS. Therefore, immuno-related toxicity assumes a potential positive prognostic role. The presence of IrAE, which derives from a hyperstimulation of the immune system, might, therefore, be specular proof of a greater anti-tumor immune response. This association has been also found in other studies $[17,18]$, but for the first time it has been documented on such a large number of patients. Moreover, although the toxicity did not replicate its statistical significance in the DSS analysis, it proved to be inversely associated to PD as the best response. Therefore, it might have a positive predictive role that requires further investigations.

Another characteristic that correlates with PD as the best response was age: older patients would seem to benefit most from the immunotherapeutic treatment. Recent metaanalyses, however, did not show a significant difference between younger and older patients $[19,20]$. Older patients might have better responses to immunotherapy because of their long-term smoking exposure and because they might benefit to a larger extent from the invigoration of the immune system. Besides a higher rate of nonsmoking, younger patients 
a Disease Specific Survival

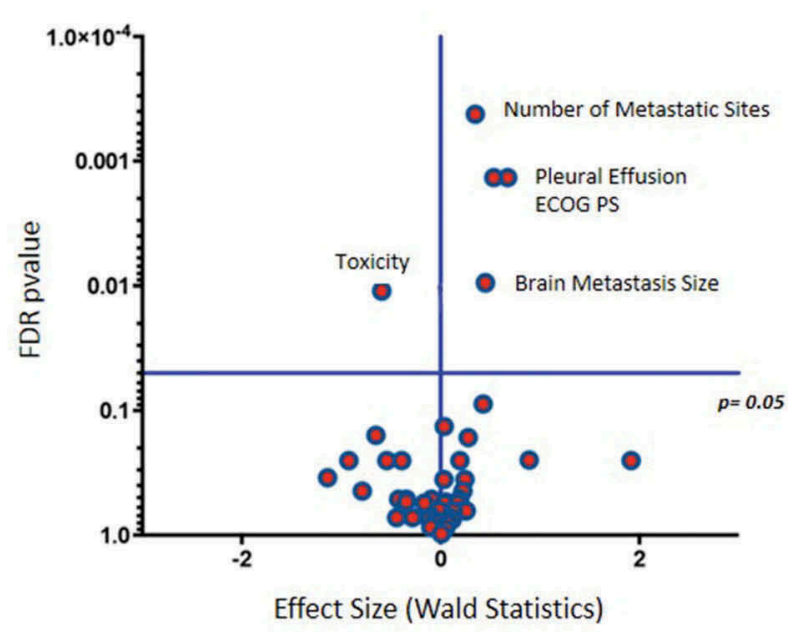

b Time to Treatment Failure

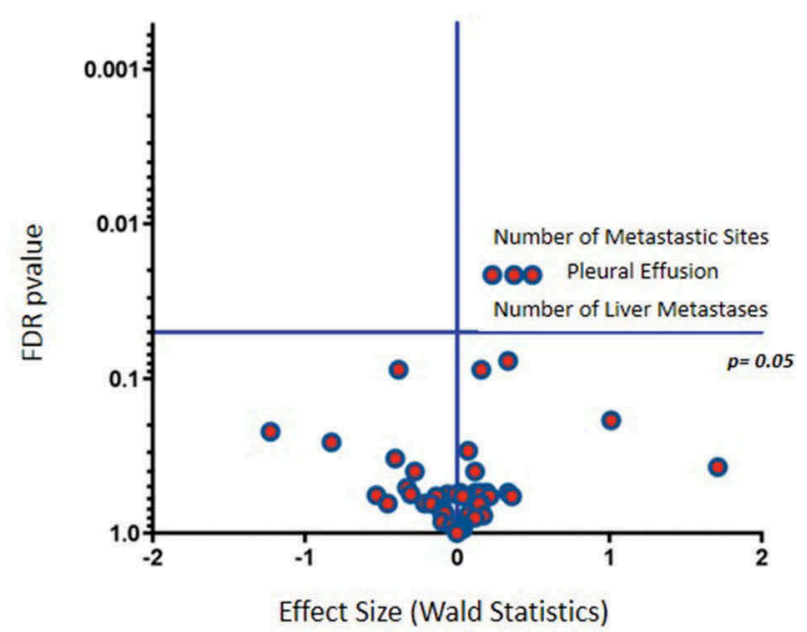

Figure 1. Prognostic factors: correlation with DSS and TTF. Cox Regression analysis.

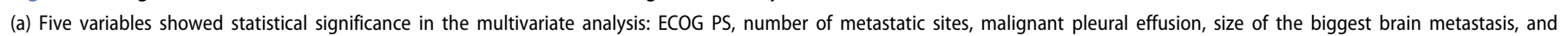
toxicity. (b) Three parameters significantly correlated with TTF: malignant pleural effusion, number of metastatic sites, and number of liver metastases.

also have a higher incidence of driver mutations, which contribute to a lower benefit from Nivolumab.

The most interesting finding of our study is that malignant pleural effusion was the only clinical parameter showing a significant correlation with $\mathrm{OR}$, as well as the only one correlating with all the endpoints of our study. Before the advent of immune-checkpoints inhibitors, survival for NSCLC patients with malignant pleural effusion was less than 6 months [21,22]. The prognostic impact of malignant pleural effusion in the era of immunotherapy is not yet known. In our study, this variable was associated both with a worse prognosis and a lower efficacy of Nivolumab. To the best of our knowledge, this is a new finding that opens up new scenarios in the field of translational research. Its magnitude led us to hypothesize that peculiar immunoregulatory mechanisms take place in the malignant pleural effusion, that hinder the immune response against cancer cells. Previous studies have documented that the pleural effusion of NSCLC patients harbors low percentages of activated CD8 + T cells and high percentages of Treg cells, especially macrophages that release cytokines and chemokines with immunosuppressive function such as IL-10 and TGF-b. In line with the 'macrophage balance hypothesis,' macrophages are capable of both promoting (M2-phenotype) and opposing tumor development (M1phenotype). Tumor-associated macrophages (TAM) in the pleural effusion (predominantly M2-phenotype) might therefore antagonize the action of immune-checkpoints inhibitors, through the systemic release of immunosuppressive cytokines [23,24].

Our study has some limitations. The entry criteria and endpoints in this exploratory analysis were dictated by the simultaneous interest in identifying clinical markers of survival and response, as well as to optimize the chances to generate novel hypotheses based on our results. Disease-Specific Survival (i.e. the time from the beginning of the treatment to cancer-associated death) is usually considered a 'soft endpoint' compared to Overall Survival (OS) (i.e. the time from the beginning of the treatment to death by any cause). As almost all patients with advanced lung cancer eventually die of the disease, we assumed competing risks to be marginal and opted for DSS, which is conceptually more appropriate for the generation of novel hypotheses on the mechanism of action and resistance to Nivolumab. The time to treatment failure (TTF) is rarely used as a surrogate endpoint of Progression-Free Survival (PFS), but is increasingly used for studies with immune-checkpoints inhibitors since PFS does not seem to capture the real benefit deriving from these agents. Furthermore, since this is a real-life study biased by retrospective design and by the different clinical practice used in the participating centers, TTF appeared as a more reliable endpoint than PFS. Finally, driver mutations and PD-L1 expression were detected only in a minority of patients (PD-L1 expression was not a mandatory test at the time the study patients were treated); therefore, the statistical analyses for these variables, whose prognostic and predictive significance during treatment with Nivolumab in NSCLC patients is still controversial, were not sufficiently powered.

\section{Conclusions}

This study identified clinical characteristics associated with efficacy of Nivolumab and survival in NSCLC patients. Among these, high tumor burden was associated with a worse prognosis and a greater probability of not responding to treatment. Immunological toxicity showed a favorable prognostic role and could represent a clinical marker of treatment efficacy. The unfavorable association between malignant pleural effusion and survival as well as to objective responses is a novel finding with important translational implications. Prospective and large-scale studies may strengthen this evidence and certify its potential clinical implications.

\section{Expert Opinion}

Nivolumab was the first PD-1 inhibitor to show survival benefit among patients with advanced NSCLC. However, although it is 
a ECOG PS

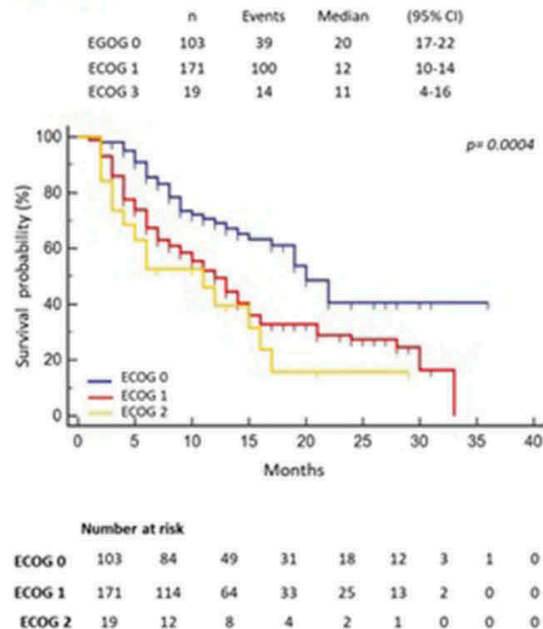

c Brain Metastasis Size
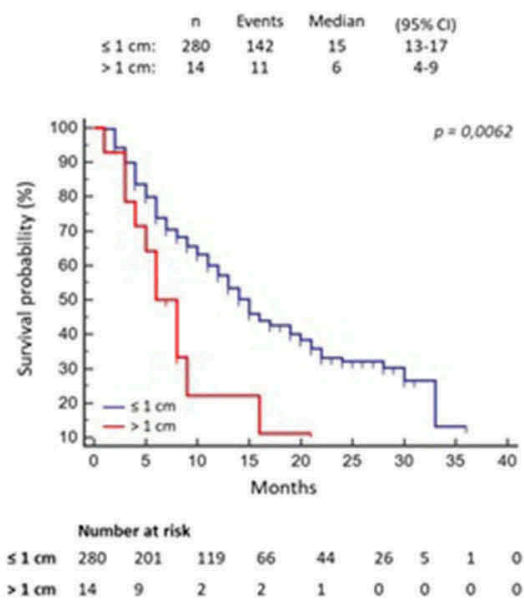

b

Number of Metastastic Sites
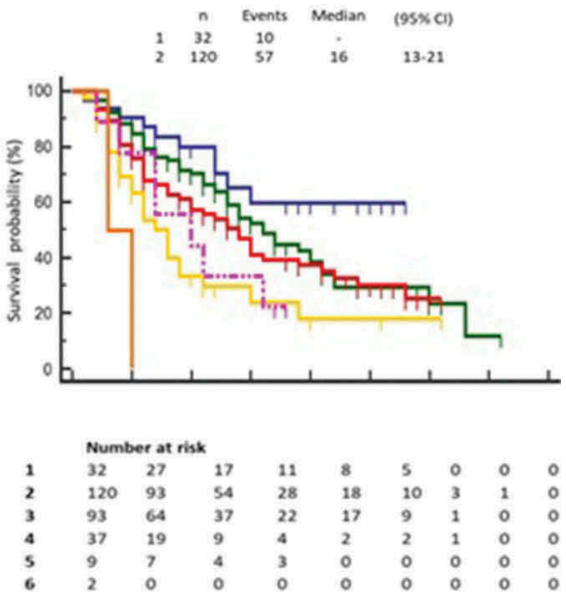

d Toxicity

$$
\begin{array}{ccccc} 
& n & \text { Events } & \text { Median } & (95 \% \mathrm{C}) \\
\text { No irAE } & 106 & 111 & 12 & 9.15 \\
\text { 2 1rAE } & 107 & 42 & 16 & 13-28
\end{array}
$$
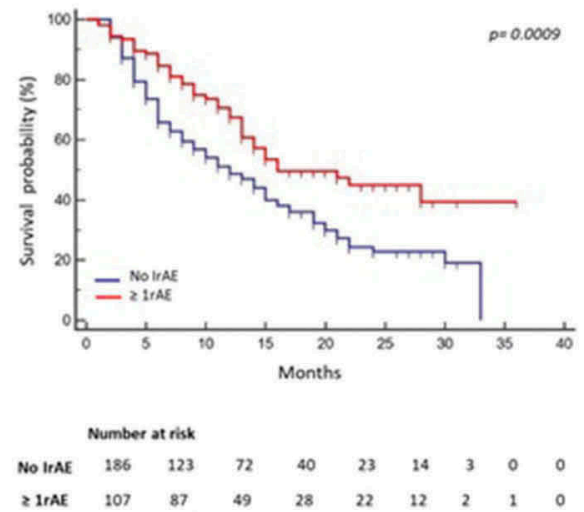

e Pleural Effusion
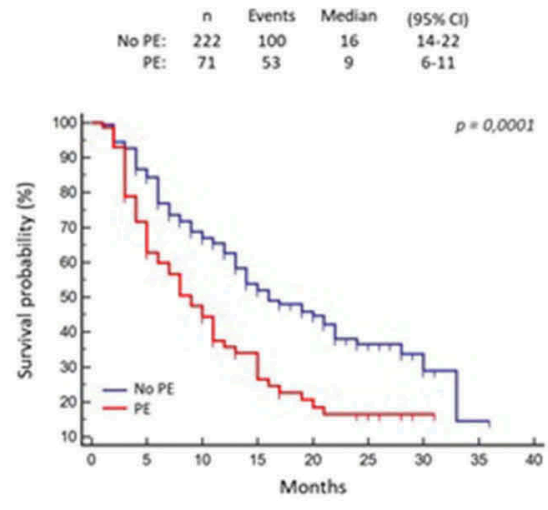

Number at risk

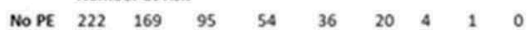

$\begin{array}{llllllllll}\text { PE } & 71 & 41 & 26 & 14 & 9 & 6 & 1 & 0 & 0\end{array}$

Figure 2. Kaplan-Meier survival curves of the variables significantly associated with DSS.

This figure shows Kaplan-Meier curves of the parameters significantly correlated with DSS. (a) Median DSS of patients with good performance status (ECOG PS 0 ) was 20 months, 8 and 9 months longer than that of patients with PS ECOG 1 and PS ECOG 2, respectively (p-value 0.0004). (b) Survival time decreases as the number of metastatic sites increases, passing from median DSS of 16 months in case of 1-2 metastatic sites to median values of only 3 months in the case of 6 sites (p-value $<0.0001$ ). (c) Median DSS among patients with brain metastases bigger than $1 \mathrm{~cm}$ (median value in study population considering $0 \mathrm{~cm}$ patients with no detectable brain metastasis) is significantly lower than among those with smaller brain metastases (m DSS 6 months versus 15 months; p-value 0.0062). (d) DSS is higher among patients with at least one immune-related adverse event than among those who have not experienced it (m DSS 16 months versus 12 months; p-value 0.0009). (e) Median DSS among patients with pleural effusion was significantly lower than among those without pleural effusion (mDSS 9 months versus 16 months; $p$-value 0.0001 ) 
a

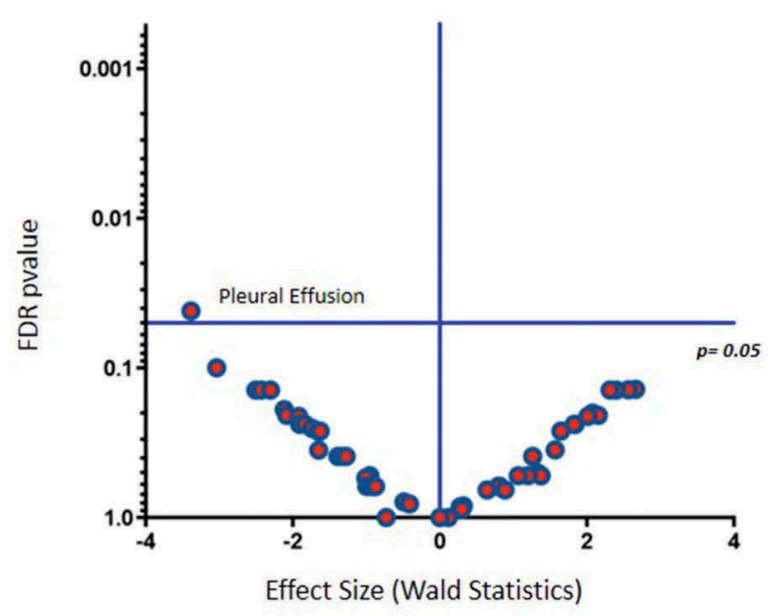

b Best Response PD

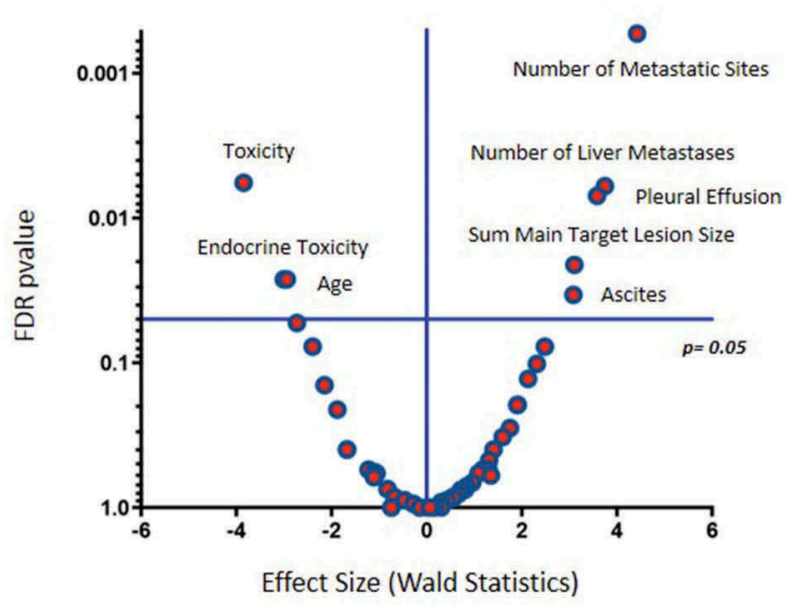

Figure 3. Predictive factors: correlation with OR (a) and PD (b). Logistic regression analysis.

(a) Pleural effusion was the only variable significantly associated with OR (p-value 0.04 ). (b) Eight variables showed a significant correlation with PD as the best response to Nivolumab treatment. Five of them correlated directly with PD: pleural effusion, number of metastatic sites, ascites, number of liver metastases, and sum of the largest diameters of the biggest target lesions in each site. The other three were inversely related to PD: toxicity (at least one IrAE), age, and endocrine toxicity.

Table 2. All factors significantly associated with the study end points.

\begin{tabular}{llcc}
\hline \multirow{2}{*}{ End point } & \multicolumn{1}{c}{ Factor } & \multicolumn{2}{c}{$\begin{array}{c}\text { Effect size } \\
\text { P-value* }\end{array}$} \\
\hline DSS & Metastatic sites number & 0.0004193 & $0,350,051$ \\
& Pleural effusion & 0.0013561 & $0,674,896$ \\
& ECOG PS & 0.0013561 & $0,535,985$ \\
& Brain metastasis lesion size & 0.0032623 & $0,452,217$ \\
TTF & Toxicity & 0.0109891 & $-0,592,607$ \\
& Liver metastasis number & 0.0214475 & $0,37,351$ \\
OR & Metastatic sites number & 0.0214475 & $0,231,529$ \\
Best response PD & Metastatic sites number & 0.0214475 & $0,495,238$ \\
& Pleural effusion & 0.00053 & $-3,383,256$ \\
& Pleural effuion & 0.0056974 & $-3,845,933$ \\
& Toxicity & 0.0060066 & $3,74,646$ \\
& Pleural effusion & 0.0070224 & $3,586,336$ \\
& Liver metastases number & 0.0209878 & $3,105,376$ \\
& Sum major target lesions size & 0.0265376 & $-3,000216$ \\
& Endocrine toxicity & 0.0265376 & $-2,939,172$ \\
& Age & 0.0339528 & 3,08718 \\
\hline
\end{tabular}

*adjusted for false discovery rate.

clear that only a subset of patients respond to immunotherapy, no reliable biomarker for prediction of the efficacy of nivolumab treatment has yet been established in daily clinical practice. Validation of clinical markers and the realization of predictive scores could optimize patient selection. This is desirable not only for Nivolumab and NSCLC, but also for the other immune-checkpoints inhibitors and for the several cancer types currently treated with immunotherapy.

\section{Author Contributions}

All authors made substantial contributions to concept and design of the study, revised the manuscript and gave their approval to the final version of the manuscript.

\section{Funding}

This paper was not funded.

\section{Declaration of interest}

The authors have no relevant affiliations or financial involvement with any organization or entity with a financial interest in or financial conflict with the subject matter or materials discussed in the manuscript. This includes employment, consultancies, honoraria, stock ownership or options, expert testimony, grants or patents received or pending, or royalties.

\section{Reviewer Disclosures}

Peer reviewers on this manuscript have no relevant financial relationships or otherwise to disclose.

\section{ORCID}

Alessio Cortellini (D) http://orcid.org/0000-0002-1209-5735

\section{References}

Papers of special note have been highlighted as either of interest $(\cdot)$ or of considerable interest $(\cdot \bullet)$ to readers.

1. Torre LA, Siegel RL, Jemal A. Lung cancer statistics. Adv Exp Med Biol. 2016;893:1-19.

2. Herzberg B, Campo MJ, Gainor JF. Immune checkpoint inhibitors in non-small cell lung cancer. Oncologist. 2017 Jan;22(1):81-88.

3. Brahmer J, Reckamp KL, Baas $P$, et al. Nivolumab versus docetaxel in advanced squamous-cell non-small-cell lung cancer. $\mathrm{N}$ Engl J Med. 2015;373:123.

- In this phase III study (CheckMate 017)y, nivolumab prolonged overall survival versus docetaxel in previously treated patients with advanced squamous NSCLC.

4. Borghaei $H$, Paz-Ares L, Horn L, et al. Nivolumab versus docetaxel in advanced nonsquamous non-small-cell lung cancer. $N$ Engl J Med. 2015;373:1627.

- In this phase III study (CheckMate 057), nivolumab showed an OS benefit versus docetaxel in previously treated patients with advanced non-squamous NSCLC.

5. Brahmer JR, Horn L, Jackman D, et al. Five-year follow-up from the CA209-003 study of Nivolumab in previously treated advanced non-small cell lung cancer: clinical characteristics of long-term 
survivors. Presented at: 2017 AACR Annual Meeting; April 1-5, 2017; Washington, DC. Abstract CT077.

6. Schadendorf D, Hodi FS, Robert C, et al. Pooled analysis of long-term survival data from phase ii and phase iii trials of ipilimumab in unresectable or metastatic melanoma. J Clin Oncol. 2015 Jun 10;33(17):1889-1894.

7. Topalian SL1, Sznol M, McDermott DF, et al. Survival, durable tumor remission, and long-term safety in patients with advanced melanomareceiving Nivolumab. J Clin Oncol. 2014 Apr 1;32(10):1020-1030.

8. Reck M, Rodríguez-Abreu D, Robinson AG, et al. Pembrolizumab versus chemotherapy for PD-L1-positive non-small-cell lung cancer. N Engl J Med. 2016 Nov 10;375(19):1823-1833.

- The KEYNOTE-024 study showed significantly longer progressionfree and overall survival with pembrolizumab monotherapy than with platinum-doublet chemotherapy in patients with a PD-L1 tumour proportion score (TPS) of $50 \%$ or greater. The results of this trial led to FDA approval of pembrolizumab monotherapy as first line treatment for patients with PD-L1 $\geq \mathbf{5 0 \%}$.

9. Herbst RS1, Baas P2, Kim DW, et al. Pembrolizumab versus docetaxel for previously treated, PD-L1-positive, advanced non-small-cell lung cancer (KEYNOTE-010): a randomised controller trial. Lancet. 2016 Apr 9;387 (10027):1540-1550.

10. Teng $F$, Meng $X$, Kong $L$, et al. Progress and challenges of predictive biomarkers of anti PD-1/PD-L1 immunotherapy: a systematic review. Cancer Lett. 2018 Feb 1;414: 166-173.

11. Chae YK, Pan A, Davis AA, et al. Biomarkers for PD-1/PD-L1 blockade therapy in non-small-cell lung cancer: is PD-L1Expression a good marker for patient selection? Clin Lung Cancer. 2016 Sep;17(5):350-361.

12. Hellmann MD, Ciuleanu TE, Pluzanski A, et al. Nivolumab plus ipilimumab in lung cancer with a high tumor mutational burden. N Engl J Med. 2018 May 31;378(22):2093-2104.

13. Gandhi L, Rodríguez-Abreu D, Gadgeel S, et al. Pembrolizumab plus chemotherapy in metastatic non-small-cell lung cancer. N Engl J Med. 2018 May 31;378(22):2078-2092.

- This phase III trial (KEYNOTE 189) showed that the addition of pembrolizumab to standard chemotherapy resulted in significantly longer overall survival and progression-free survival than chemotherapy alone, in patients with previously untreated metastatic nonsquamous NSCLC.

14. Paz-Ares L, Luft A, Vicente $D$, et al. Pembrolizumab plus chemotherapy for squamous non-small-cell lung cancer. N Engl J Med. 2018;379:2040-2051.
- This phase III trial (KEYNOTE 407) showed that the addition of pembrolizumab to standard chemotherapy in patients with previously untreated metastatic squamous NSCLC resulted in significantly longer overall survival and progression-free survival, than chemotherapy alone, regardless of the level of PDL1 expression.

15. Kaplan EL, Meier P. Nonparametric estimation from incomplete observations. J Am Stat Assoc. 1958;53:457-481.

16. Peto R, Pike MC, Armitage $P$, et al. Design and analysis of randomized clinical trials requiring prolonged observation of each patient. II. analysis and examples. $\mathrm{Br} J$ Cancer. 1977;35:1-39.

17. Sato K, Akamatsu H, Murakami E, et al. Correlation between immune-related adverse events and efficacy in non-small cell lung cancer treated with Nivolumab. Lung Cancer. 2018;115:71-74.

18. Tereatoka S, Fujimoto D, Morimoto T, et al. Early immune-related adverse events and association with outcome in advanced non-small cell lung cancer patients treated with Nivolumab: a prospective cohort study. J Thorac Oncol. 2017;12 (12):1798-1805.

19. Elias R, Giobbie-Hurder A, McCleary NJ. Efficacy of PD-1 \& PD-L1 inhibitors in older adults: a meta-analysis. J Immunother Cancer. 2018;6:26.

20. Nishijima TF, Muss HB, Shachar SS, et al. Comparison of efficacy of immune checkpoint inhibitors ( $\mathrm{ICls})$ between younger and older patients: a systematic review and meta-analysis. Cancer Treat Rev. 2016;45:30-37.

21. Morgensztern D1, Waqar S, Subramanian J, et al. Prognostic impact of malignant pleural effusion at presentation in patients with metastatic non-small-cell lung cancer. J Thorac Oncol. 2012 Oct;7 (10):1485-1489.

22. Sugiura $S$, Ando $Y$, Minami $H$, et al. Prognostic value of pleural effusion in patients with non-small cell lung cancer. Clin Cancer Res. 1997 Jan;3(1):47-50.

23. DeLong $P$, Carroll RG, Henry AC, et al. Regulatory $T$ cells and cytokines in malignant pleural effusions secondary to mesothelioma and carcinoma. Cancer Biol Ther. 2005;4(3): 342-346.

24. Quatromoni JG, Eruslanov E. Tumor-associated macrophages: function, phenotype, and link to prognosis in human lung cancer. Am J Transl Res. 2012;4(4):376-389. 